\title{
Seletividade de Herbicidas Aplicados em Pré-Emergência da Cultura da CANa-De-AÇÚCAR (RB 867515) TRATADA COM NEMATICIDAS ${ }^{1}$
}

\author{
Selectivity of Herbicides Applied on Pre-Emergence of Nematicide-treated Sugarcane Crop \\ (RB 867515)
}

BARELA, J.F. ${ }^{2}$ e CHRISTOFFOLETI, P.J. ${ }^{3}$

\begin{abstract}
RESUMO - A aplicação simultânea de duas ou mais classes de defensivos sobre uma cultura agrícola pode provocar toxicidade às plantas. Assim, conduziu-se um experimento com o objetivo de avaliar a seletividade de herbicidas à cultura da cana-de-açúcar (RB 867515), quando aplicados em condição de pré-emergência, em áreas previamente tratadas com nematicidas no sulco de plantio. O experimento foi desenvolvido em área comercial de produção de cana-de-açúcar, no município de Piracicaba - SP, entre abril de 2003 e julho de 2004. Os tratamentos resultaram da combinação entre nove fatores herbicidas e quatro fatores nematicidas. Os herbicidas usados no experimento foram: sulfentrazone, tebuthiuron, metribuzin, ametrina, diuron, clomazone, pendimethalin e diuron + hexazinone, além de uma testemunha capinada. Os nematicidas utilizados foram: carbofuran, terbufós, aldicarb e uma testemunha sem nematicida. Avaliou-se a fitotoxicidade das diferentes combinações aos 15, 30, 60 e 90 dias após a brotação, o rendimento (t ha ${ }^{-1}$ ) e os parâmetros tecnológicos qualitativos. Observou-se que a seletividade inicial dos herbicidas foi prejudicada em função da interação das diferentes classes de defensivos utilizadas. Os sintomas de fitotoxicidade foram agudos, e os casos mais evidentes se originaram da associação dos nematicidas com clomazone, pendimethalin e tebuthiuron. Contudo, os danos fitotóxicos não se refletiram em perdas significativas de rendimento ou de qualidade de colmos, fato esse que pode ter sido influenciado pela capacidade de recuperação de injúrias apresentada pela variedade $\mathrm{RB} 867515$, pela ocorrência de altas infestações de nematóides ou pela elevada variabilidade experimental.
\end{abstract}

Palavras-chave: cana-de-açúcar, manejo, defensivos, interações.

ABSTRACT - Simultaneous applications of two or more classes of herbicides on a crop may promote ,totoxicity in the plants. Therefore, an experiment was conducted to evaluate herbicide selectivity in sugarcane, when applied under pre-emergence condition, in areas previously treated with nematicides on the planting furrow. The experiment was carried out in a commercial sugarcane area in Piracicaba (SP), from April 2003 to July 2004. The treatments resulted from the combination of nine herbicide factors and four nematicide factors. The herbicides used in the experiment were: sulfentrazone, tebuthiuron, metribuzin, ametrina, diuron, clomazone, pendimethalin and diuronthexazinone, besides a hand-weeded check. The nematicides used were: carbofuran, terbufos, aldicarb and a check without nematicide. Phytotoxicity of the different combinations at 15, 30, 60 and 90 days after sprouting, yield $\left(t h a^{-1}\right)$ and qualitative technological parameters were evaluated. It was observed that the initial selectivity of the herbicides was reduced as a function of the interaction between the different classes used. Phytotoxic symptoms were acute, with the most serious cases originating from the association of nematicides with clomazone, pendimethalin and tebuthiuron. However, phytotoxic damages did not reflect a significant yield or qualitative losses, a fact that may have influenced injury recovery capacity, as presented by the RB 867515 variety, due to high nematode infestations or high experimental variability.

Keywords: sugarcane, management, herbicides, interactions.

Recebido para publicação em 23.11.2005 e na forma revisada em 5.5.2006.

2 Eng.-Agr., M.S. <jfbarela@ terra.com.br>; ${ }^{3}$ Professor Associado do Departamento de Produção Vegetal da Escola Superior de Agricultura "Luiz de Queiroz" - ESALQ/USP, Caixa Postal 09, 13419-900 Piracicaba-SP, <pjchrist@esalq.usp.br>. 


\section{INTRODUÇÃO}

A cana-de-açúcar (Saccharum officinarum) é uma das culturas mais importantes para o Brasil, pois tem exercido uma função duplamente destacável. Por um lado, a produção açucareira fundamenta grande parte das divisas do país, que tem se apresentado como o maior exportador mundial de açúcar, com cerca de 6 milhões de toneladas (Procópio et al., 2004); por outro, a produção de álcool constitui importante alternativa brasileira à substituição de determinados derivados do petróleo. Atualmente, a cultura da cana-de-açúcar ocupa uma área superior a 5,0 milhões de hectares e apresenta produtividade média em torno de 73,4 t ha-1 (Negrisoli, 2001).

A produtividade média brasileira poderia ser maior se o desenvolvimento da cultura não fosse prejudicado por fatores bióticos e abióticos (clima e solo). Dentre os fatores bióticos, a incidência de plantas daninhas e a ocorrência de nematóides contribuem para o declínio acentuado da produção. Em áreas infestadas com nematóides, as perdas podem chegar a mais de 50\%, no caso das espécies Meloidogyne incognita e Pratylenchus zeae (Novaretti, 1981; Dinardo-Miranda \& Ferraz, 1991). No entanto, a presença de plantas daninhas pode causar reduções na quantidade e na qualidade do produto colhido e diminuir o número de cortes viáveis, além de aumentar os custos em cerca de 30\% para cana-soca e de 15 a 20\% para cana-planta (Lorenzi, 1988, 1995; Procópio et al., 2004).

Na cultura da cana-de-açúcar, o controle químico (nematicidas e herbicidas) tem contribuído significativamente para manutenção da produtividade agrícola, mesmo em áreas problemáticas. A aplicação sistemática de nematicidas no sulco de plantio e, logo após, a pulverização de herbicidas em condição de pré-emergência, tanto das plantas daninhas quanto da cultura, ocorrem de forma rotineira nas áreas produtoras. Quando as práticas agrícolas recomendadas são seguidas, esses compostos químicos não são prejudiciais individualmente, porém a tolerância das plantas a um determinado defensivo pode ser alterada pela presença de outro. Uma cultura tolerante a um herbicida pode ter essa capacidade reduzida devido à interação sinérgica entre o herbicida e um outro produto químico aplicado, como um nematicida, por exemplo (DinardoMiranda et al., 2001a).

$\mathrm{Na}$ literatura existem alguns casos de interações sinérgicas que envolvem essas classes de defensivos nas culturas do milho e da soja. As interações promovem a redução do comprimento de radículas, diminuição da respiração de plântulas, redução da capacidade de tolerância de certos herbicidas, redução da fotossíntese líquida, diminuição da efetividade de enzimas vitais, entre outros (Ahrens, 1990; López-Ovejero et al., 2003). Diversos pesquisadores, entre eles Blanco et al. (1980), observaram problemas de fitotoxicidade na cultura da cana-de-açúcar devido à aplicação seqüencial de um nematicida e um herbicida. Os principais sintomas verificados foram: clorose acentuada ao longo do limbo foliar, acompanhada de secamento das folhas a partir do ápice e da margem, atingindo inclusive o grau de paralisação do crescimento das plantas; diminuição da altura; perda de rendimento de sacarose com a diminuição da espessura de colmos; menor número de plantas por metro de plantio, entre outros.

Tendo em vista essas observações, associado à necessidade de maiores informações que englobem os atuais produtos disponiveis no mercado, tem-se a necessidade de trabalhos que possam servir de base para as recomendações agrícolas do setor produtivo. Assim, conduziu-se um experimento com o objetivo de avaliar a seletividade de herbicidas à cultura da cana-de-açúcar quando aplicados em condição de pré-emergência sobre solo previamente tratado com nematicidas.

\section{MATERIAL E MÉTODOS}

Este trabalho foi desenvolvido em campo, em área comercial produtora de cana-deaçúcar pertencente à Usina Santa Helena (Grupo COSAN), localizada no município de

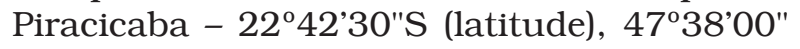
W (longitude) e $560 \mathrm{~m}$ de altitude, no Estado de São Paulo, entre abril de 2003 e julho de 2004. Segundo Köeppen (1948), o clima da região é do tipo Cwa, ou seja, mesotérmico, tropical úmido, com três meses mais secos (junho, julho e agosto) e com concentração de chuvas no verão. 
O solo da área experimental é do tipo Latossolo Vermelho-Amarelo distrófico (LVd), álico, de textura arenosa e com as propriedades químicas apresentadas na Tabela 1 . O solo foi previamente preparado por meio de uma aração a $0,40 \mathrm{~m}$ de profundidade, seguida por duas gradagens, em área de reforma de canavial. Para a correção do solo foram aplicadas 4,5 t ha $^{-1}$ de calcário dolomítico $(30 \% \mathrm{CaO}$ e $18 \%$ $\mathrm{MgO}$ ) com poder de reação e neutralização total (PRNT) de 68\%. No plantio da cana-de-açúcar, a adubação mineral constou de $450 \mathrm{~kg} \mathrm{ha}^{-1} \mathrm{da}$ fórmula comercial 04-30-10 (N-P-K ).

O experimento foi instalado no dia 3/4/ 2003. Os sulcos de plantio foram abertos com cerca de 0,40 m de profundidade e espaçados de $1,40 \mathrm{~m}$. A variedade utilizada foi RB 867515, depositando-se uma média de 15 gemas por metro de sulco. Durante o experimento, foram realizadas duas amostragens de raízes de cana-de-açúcar e do solo da área, com o objetivo de monitorar os níveis populacionais de nematóides.

Os tratamentos resultaram da combinação entre quatro níveis do fator nematicidas (g ha-1): terbufós (3.000), carbofuran (2.950), aldicarb (1.500) e testemunha sem aplicação; e nove niveis do fator herbicidas ( $\mathrm{g} \mathrm{ha}^{-1}$ ): ametrina (2.500), clomazone (1.000), diuron (2.250), diuron + hexazinone $(1.066+134)$, metribuzin (1.440), pendimethalin (1.750), sulfentrazone (800), tebuthiuron (900) e parcelas testemunhas sem a aplicação de herbicidas. Todo o experimento foi mantido sem a presença de plantas daninhas, por meio de capinas manuais. Os tratamentos herbicidas e nematicidas estão adequadamente descritos na Tabela 2 .

Tabela 1 - Análise química do solo da área experimental. Piracicaba, 2003/04

\begin{tabular}{|c|c|c|c|c|c|c|c|c|c|c|c|c|}
\hline $\mathrm{pH}$ & $\mathrm{MO}$ & $\mathrm{P}$ & $\mathrm{S}$ & $\mathrm{K}$ & $\mathrm{Ca}$ & $\mathrm{Mg}$ & $\mathrm{Al}$ & $\mathrm{H}+\mathrm{Al}$ & $\mathrm{SB}$ & $\mathrm{T}$ & $\mathrm{V}$ & $\mathrm{m}$ \\
\hline$\left(\mathrm{CaCl}_{2}\right)$ & $\left(\mathrm{g} \mathrm{dm}^{-3}\right)$ & \multicolumn{1}{|c|}{$\left(\mathrm{mg} \mathrm{dm}^{-3}\right)$} & \multicolumn{1}{|c|}{$\left(\mathrm{mmol}_{\mathrm{c}} \mathrm{dm}^{-3}\right)$} & $(\%)$ \\
\hline 4,0 & 13,0 & 4,0 & 7,0 & 0,1 & 3,0 & 3,0 & 12,0 & 47,0 & 6,1 & 53,1 & 11 & 22,5 \\
\hline
\end{tabular}

Fonte: dados cedidos pela Usina Santa Helena (Cosan). Métodos de extração: pH: Sol. $\mathrm{CaCl}_{2}$; MO: ácido sulfúrico; P, $\mathrm{K}, \mathrm{Ca}, \mathrm{Mg}: \mathrm{resina}$; $\mathrm{H}+\mathrm{Al}$ : tampão SMP; Al, KCl, S: fosf. cálcio.

Tabela 2 - Tratamentos herbicidas e nematicidas utilizados no experimento. Piracicaba, 2003/04

\begin{tabular}{|c|c|c|c|c|}
\hline \multicolumn{3}{|c|}{ Herbicida } & \multicolumn{2}{|c|}{ Dose } \\
\hline \multirow{2}{*}{ Nome comum } & \multirow{2}{*}{ Nome comercial } & \multirow{2}{*}{ Formulação $0^{\underline{1}}$} & i.a. ${ }^{2 /}$ & p.c. ${ }^{3 /}$ \\
\hline & & & $\left(\mathrm{g} \mathrm{ha}^{-1}\right)$ & $\left(\mathrm{L} \mathrm{ou} \mathrm{kg} \mathrm{ha}{ }^{-1}\right)$ \\
\hline 1. ametrina & Herbipak 500 BR & $\mathrm{SC}$ & 2.500 & 5,0 \\
\hline 2. clomazone & Gamit & $\mathrm{CE}$ & 1.000 & 2,0 \\
\hline 3. diuron & Cention SC & SC & 2.250 & 4,5 \\
\hline 4. diuron + hexazinone & Advance & GRDA & $1.066+134$ & 2,0 \\
\hline 5. metribuzin & Sencor 480 & SC & 1.440 & 3,0 \\
\hline 6. pendimethalin & Herbadox $500 \mathrm{CE}$ & $\mathrm{CE}$ & 1.750 & 3,5 \\
\hline 7. sulfentrazone & Boral 500 SC & SC & 800 & 1,6 \\
\hline 8. tebuthiuron & Combine $500 \mathrm{SC}$ & SC & 900 & 1,9 \\
\hline 9. testemunha & - & - & - & - \\
\hline \multicolumn{3}{|c|}{ Nematicida } & & \\
\hline 1. terbufos & Counter $150 \mathrm{G}$ & $\mathrm{G}$ & 3.000 & 20,0 \\
\hline 2. carbofuran & Furadan 350 SC & SC & 2.950 & 8,5 \\
\hline 3. aldicarb & Temik 150 & $\mathrm{G}$ & 1.500 & 10,0 \\
\hline 4. testemunha & - & - & - & - \\
\hline
\end{tabular}

${ }^{1}$ SC - suspensão concentrada, CE - concentrado emulsionável, GRDA - grânulos dispersíveis em água e G - grânulos; ${ }^{2}$ ingrediente ativo; ${ }^{3}$ produto comercial. 
Os nematicidas foram aplicados momentos antes do fechamento dos sulcos, com auxílio de uma granuladeira tratorizada. Cada nematicida foi aplicado em quatro faixas de $63 \mathrm{~m}$ de comprimento e em 10 linhas de plantio de forma alternada. Além disso, quatro faixas com as mesmas áreas foram deixadas sem aplicação de nematicidas, para servirem como testemunhas. Depois que os sulcos foram fechados, todas as faixas foram divididas em nove subparcelas com $7 \mathrm{~m}$ de comprimento e dez linhas de cana-de-açúcar. A área útil das subparcelas restringiu-se às oito linhas centrais de cana-de-açúcar, desprezando-se $1 \mathrm{~m}$ de bordadura ao início e ao término da subparcela.

Os herbicidas foram aplicados no dia $8 / 4 / 2003$, em total pré-emergência das plantas daninhas e da cultura. Para isso, utilizou-se um pulverizador costal pressurizado por $\mathrm{CO}_{2}$, à pressão constante de $2 \mathrm{kgf} \mathrm{cm}^{-2}$, com barra equipada com quatro pontas do tipo leque Teejet 110.02 , espaçados em $0,50 \mathrm{~m}$ e volume equivalente de calda da ordem de $200 \mathrm{~L} \mathrm{ha}^{-1}$. No momento das aplicações, a velocidade do vento, a UR e a temperatura oscilaram entre 4,0 e $5,5 \mathrm{~km} \mathrm{~h}^{-1}, 67,3$ e $72,1 \%$ e 24,2 e $25,3{ }^{\circ} \mathrm{C}$, respectivamente.

O delineamento experimental adotado foi do tipo parcelas subdivididas com quatro repetições, em que os nematicidas foram alocados no âmbito das parcelas e os herbicidas nas subparcelas, o que correspondeu a um total de 144 subparcelas e uma área experimental de 1,41 ha. As variáveis avaliadas foram: fitotoxicidade aos 15, 30, 60 e 90 dias após brotação (DAB), rendimento ( $\mathrm{t} \mathrm{ha}^{-1}$ ) e parâmetros tecnológicos (graus brix, pol., fibra e ATR). A data de brotação foi fixada quando $50 \%$ das plantas apresentavam-se nesse estádio. As notas de fitotoxicidade foram administradas em intervalo compreendido entre 0 (ausência de sintomas) e 100 (morte das plantas), conforme metodologia proposta por Velini et al. (1995).

O rendimento foi obtido após a queima da área, por meio da pesagem do material vegetal presente na área útil das subparcelas, no dia $30 / 7 / 2004$. Os dados obtidos foram submetidos à análise de variância (teste F), e as médias, comparadas por meio do teste de Tukey com nível de significância de 5\%.

\section{RESULTADOS E DISCUSSÃO}

No início de desenvolvimento da cultura, as plantas de cana-de-açúcar das parcelas que receberam os tratamentos envolvendo herbicidas demonstraram sintomas de fitotoxicidade, como clorose, necroses e falhas visuais de brotação. Blanco et al. (1980) descreveram esses sintomas como amarelecimento do limbo foliar, seguido pela requeima das folhas, começando pelo ápice e pelas laterais e estendendo-se para a nervura central, ocorrendo, em alguns casos, secamento total das folhas.

A primeira avaliação visual da fitotoxicidade foi feita aos $15 \mathrm{DAB}$ e as médias estão apresentadas na Tabela 3. As injúrias mostraram-se bastante severas: as plantas de todos os tratamentos, com exceção das testemunhas sem herbicidas, apresentaram sintomas de dano. Nessa época, pôde-se perceber que a aplicação conjunta de aldicarb + pendimethalin $(26,25 \%)$, carbofuran + clomazone $(26,25 \%)$ e terbufós + clomazone $(38,75 \%)$ provocou os sintomas mais elevados de toxicidade às plantas. Isso indica a ocorrência de um acréscimo nas injúrias (efeito sinergístico) dos herbicidas, causado pelo uso dos nematicidas no sulco de plantio. Nas parcelas onde foram aplicados aldicarb, carbofuran e terbufós, os incrementos de fitotoxicidade foram de 11,25, 8,75 e 21,25\%, respectivamente, quando comparados às parcelas onde somente foram aplicados os herbicidas. Dinardo-Miranda et al. (2001b) observaram que as plantas das parcelas que receberam terbufós + clomazone também apresentaram maior grau de fitotoxicidade inicial.

Quando apenas os herbicidas são analisados, fica evidente que o potencial de fitointoxicação ou o grau de seletividade para a cultura da cana-de-açúcar de cada um deles é alterado pela presença do nematicida no sulco. Todos os herbicidas estudados foram menos seletivos à cultura quando na presença de algum nematicida, porém os graus da interação são variáveis. O herbicida clomazone, por exemplo, chega a ser 21,25\% menos seletivo quando associado ao terbufós; já o herbicida diuron é apenas 5\% mais fitotóxico na presença do mesmo nematicida. 
Aos $30 \mathrm{DAB}$, os tratamentos citados continuaram como os mais danosos às plantas, mas com menor diferença em relação à testemunha. Os tratamentos aldicarb + pendimethalin e carbofuran + clomazone causaram 10 e 6,25\% mais injúrias. Por sua vez, o tratamento terbufós + clomazone proporcionou incremento de $23,75 \%$ de fitotoxicidade em relação ao tratamento em que somente foi aplicado o clomazone (Tabela 4).

Esses dados estão em concordância com os trabalhos de Dinardo-Miranda et al. (2001a, b), que também não relataram sintomas severos de toxicidade nas plantas que receberam o tratamento carbofuran + clomazone, em contraposição ao tratamento de terbufós + clomazone, em que os sintomas foram detectados.

Aos 60 DAB (Tabela 5), os mesmos tratamentos continuaram como os mais prejudiciais à cultura da cana-de-açúcar, porém suas diferenças em relação à testemunha diminuíram com o decorrer das avaliações. Aos 90 DAB, o tratamento aldicarb + tebuthiuron apresentou a maior porcentagem de fitotoxicidez, superando os danos provocados pelo tratamento aldicarb + pendimethalin (Tabela 6).

Vários fatores afetam a interação potencial entre duas classes de defensivos, e um dos mais importantes é como estes são absorvidos e translocados dentro da planta (Hartzler et al., 2003); todavia, os mecanismos de seletividade também são fundamentais. Uma explicação para a interação entre nematicidas e herbicidas está relacionada com a ação inibitória dos metabólitos do terbufós sobre o citocromo P-450, responsável pela metabolização de diversos herbicidas em plantas. Sem a atividade metabólica plena, os herbicidas se acumulam, provocando injúrias (Baerg \& Barrett, 1993; Siminszky et al., 1995; Hinz et al., 1997).

Tabela 3 - Fitotoxicidade (\%) observada na interação dos tratamentos herbicidas e nematicidas aos 15 dias após brotação da canade-açúcar. Piracicaba, 2003/04

\begin{tabular}{|c|c|c|c|c|}
\hline \multirow{2}{*}{ Herbicida $^{\underline{1}}$} & \multicolumn{4}{|c|}{ Nematicida $^{1 /}$} \\
\hline & Testemunha & Aldicarb & Carbofuran & Terbufós \\
\hline Testemunha & $0,00 \mathrm{~A} \mathrm{a}$ & $0,00 \mathrm{~A} \mathrm{a}$ & $0,00 \mathrm{~A} \mathrm{a}$ & $0,00 \mathrm{~A} \mathrm{a}$ \\
\hline Tebuthiuron & $16,25 \mathrm{C} \mathrm{a}$ & $25,00 \mathrm{D} \mathrm{b}$ & $25,00 \mathrm{CD} \mathrm{b}$ & $28,75 \mathrm{C} \mathrm{b}$ \\
\hline Sulfentrazone & $8,75 \mathrm{~B} \mathrm{a}$ & $15,00 \mathrm{~B} \mathrm{~b}$ & $18,75 \mathrm{BC} \mathrm{b}$ & $18,75 \mathrm{~B} \mathrm{~b}$ \\
\hline Pendimethalin & $15,00 \mathrm{BC} \mathrm{a}$ & $26,25 \mathrm{D}$ bc & $23,75 \mathrm{BCD} \mathrm{b}$ & $30,00 \mathrm{Cc}$ \\
\hline Metribuzin & $12,50 \mathrm{BC} \mathrm{a}$ & $17,50 \mathrm{BC} \mathrm{ab}$ & $18,75 \mathrm{BC} \mathrm{b}$ & $20,00 \mathrm{~B} \mathrm{~b}$ \\
\hline Diuron+hexazinone & $12,50 \mathrm{BC} \mathrm{a}$ & $17,50 \mathrm{BC}$ ab & $17,50 \mathrm{~B} \mathrm{ab}$ & $20,00 \mathrm{~B} \mathrm{~b}$ \\
\hline Diuron & $13,75 \mathrm{BC} \mathrm{a}$ & $17,50 \mathrm{BC} \mathrm{a}$ & $18,75 \mathrm{BC} \mathrm{a}$ & $18,75 \mathrm{~B} \mathrm{a}$ \\
\hline Clomazone & $17,50 \mathrm{C} \mathrm{a}$ & $22,50 \mathrm{CD} a b$ & $26,25 \mathrm{D} \mathrm{b}$ & $38,75 \mathrm{Dc}$ \\
\hline \multirow[t]{2}{*}{ Ametrina } & $8,75 \mathrm{~B} \mathrm{a}$ & $17,50 \mathrm{BC} \mathrm{b}$ & $18,75 \mathrm{BC} \mathrm{b}$ & $21,25 \mathrm{~B} \mathrm{~b}$ \\
\hline & \multicolumn{2}{|c|}{$\mathrm{DMS}_{\text {(coluna) }}=6,26$} & \multicolumn{2}{|c|}{$\mathrm{DMS}_{(\text {linha) }}=5,16$} \\
\hline
\end{tabular}

1 Médias seguidas de mesma letra, maiúscula na coluna e minúscula na linha, não diferem entre si pelo teste de Tukey a 5\% de significância.

Tabela 4 - Fitotoxicidade (\%) observada na interação dos tratamentos herbicidas e nematicidas aos 30 dias após brotação da cana-de-açúcar. Piracicaba, 2003/04

\begin{tabular}{|c|c|c|c|c|}
\hline \multirow{2}{*}{ Herbicida $^{1 /}$} & \multicolumn{4}{|c|}{ Nematicida $^{1 /}$} \\
\hline & Testemunha & Aldicarb & Carbofuran & Terbufós \\
\hline Testemunha & $0,00 \mathrm{~A} \mathrm{a}$ & $0,00 \mathrm{~A} \mathrm{a}$ & $0,00 \mathrm{~A} \mathrm{a}$ & $0,00 \mathrm{~A} \mathrm{a}$ \\
\hline Tebuthiuron & $11,25 \mathrm{CD}$ a & $16,25 \mathrm{BC} \mathrm{b}$ & $16,25 \mathrm{BC} \mathrm{b}$ & $21,25 \mathrm{C} \mathrm{c}$ \\
\hline Sulfentrazone & $5,00 \mathrm{AB}$ a & $13,75 \mathrm{~B} \mathrm{~b}$ & $13,75 \mathrm{BC} \mathrm{b}$ & $15,00 \mathrm{~B} \mathrm{~b}$ \\
\hline Pendimethalin & $11,25 \mathrm{CD}$ a & $21,25 \mathrm{C} \mathrm{c}$ & $16,25 \mathrm{BC} \mathrm{b}$ & $25,00 \mathrm{C} \mathrm{c}$ \\
\hline Metribuzin & $11,25 \mathrm{CD}$ a & $15,00 \mathrm{~B}$ a & $13,75 \mathrm{BC} \mathrm{a}$ & $11,25 \mathrm{~B} \mathrm{a}$ \\
\hline Diuron+hexazinone & $10,00 \mathrm{BCD}$ a & $13,75 \mathrm{~B} \mathrm{ab}$ & $12,50 \mathrm{~B} \mathrm{ab}$ & $15,00 \mathrm{~B} \mathrm{~b}$ \\
\hline Diuron & $10,00 \mathrm{BCD}$ a & $13,75 \mathrm{~B} \mathrm{ab}$ & $13,75 \mathrm{BC}$ ab & $15,00 \mathrm{~B} \mathrm{~b}$ \\
\hline Clomazone & $12,50 \mathrm{D}$ a & $16,25 \mathrm{BC}$ ab & $18,75 \mathrm{C} \mathrm{b}$ & $36,25 \mathrm{D} \mathrm{c}$ \\
\hline \multirow[t]{2}{*}{ Ametrina } & $6,25 \mathrm{BC} \mathrm{a}$ & $15,00 \mathrm{~B} \mathrm{~b}$ & $13,75 \mathrm{BC} \mathrm{b}$ & $15,00 \mathrm{~B} \mathrm{~b}$ \\
\hline & \multicolumn{2}{|c|}{$\mathrm{DMS}_{\text {(coluna) }}=5,30$} & \multicolumn{2}{|c|}{$\mathrm{DMS}_{\text {(linha) }}=4,37$} \\
\hline
\end{tabular}

1/ Médias seguidas de mesma letra, maiúscula na coluna e minúscula na linha, não diferem entre si pelo teste de Tukey a 5\% de significância. 
Tabela 5 - Fitotoxicidade (\%) observada na interação dos tratamentos herbicidas e nematicidas aos 60 dias após brotação da cana-de-açúcar. Piracicaba, 2003/04

\begin{tabular}{|c|c|c|c|c|}
\hline \multirow{2}{*}{ Herbicida ${ }^{1 /}$} & \multicolumn{4}{|c|}{ Nematicida $^{1 /}$} \\
\hline & Testemunha & Aldicarb & Carbofuran & Terbufós \\
\hline Testemunha & $0,00 \mathrm{~A} \mathrm{a}$ & $0,00 \mathrm{~A} \mathrm{a}$ & $0,00 \mathrm{~A} \mathrm{a}$ & $0,00 \mathrm{~A} \mathrm{a}$ \\
\hline Tebuthiuron & $5,00 \mathrm{~B} \mathrm{a}$ & $5,00 \mathrm{~B} \mathrm{a}$ & $5,00 \mathrm{~B} \mathrm{a}$ & $10,00 \mathrm{BC} \mathrm{b}$ \\
\hline Sulfentrazone & $1,25 \mathrm{AB}$ a & $5,00 \mathrm{~B} \mathrm{a}$ & $3,75 \mathrm{AB}$ a & $10,00 \mathrm{BC} \mathrm{b}$ \\
\hline Pendimethalin & $3,75 \mathrm{AB}$ a & $5,00 \mathrm{~B} \mathrm{a}$ & $3,75 \mathrm{AB}$ a & $10,00 \mathrm{BC} \mathrm{b}$ \\
\hline Metribuzin & $2,50 \mathrm{AB}$ a & $5,00 \mathrm{~B} \mathrm{ab}$ & $5,00 \mathrm{~B} \mathrm{ab}$ & $8,75 \mathrm{~B} \mathrm{~b}$ \\
\hline Diuron+hexazinone & $3,75 \mathrm{AB}$ a & $6,25 \mathrm{~B} \mathrm{ab}$ & $5,00 \mathrm{~B} \mathrm{a}$ & $10,00 \mathrm{BC} \mathrm{b}$ \\
\hline Diuron & $2,50 \mathrm{AB}$ a & $5,00 \mathrm{~B} \mathrm{a}$ & $5,00 \mathrm{~B} \mathrm{a}$ & $10,00 \mathrm{BC} \mathrm{b}$ \\
\hline Clomazone & $3,75 \mathrm{AB}$ a & $6,25 \mathrm{~B}$ a & $5,00 \mathrm{~B} \mathrm{a}$ & $13,75 \mathrm{C} \mathrm{b}$ \\
\hline \multirow[t]{2}{*}{ Ametrina } & $1,25 \mathrm{AB}$ a & $3,75 \mathrm{AB}$ a & $5,00 \mathrm{~B} \mathrm{a}$ & $11,25 \mathrm{BC} \mathrm{b}$ \\
\hline & \multicolumn{2}{|c|}{$\mathrm{DMS}_{\text {(coluna) }}=4,59$} & \multicolumn{2}{|c|}{$\mathrm{DMS}_{(\text {linha) }}=3,78$} \\
\hline
\end{tabular}

1/ Médias seguidas de mesma letra, maiúscula na coluna e minúscula na linha, não diferem entre si pelo teste de Tukey a 5\% de significância.

Tabela 6 - Fitotoxicidade (\%) observada na interação dos tratamentos herbicidas e nematicidas aos 90 dias após brotação da cana-de-açúcar. Piracicaba, 2003/04

\begin{tabular}{|c|c|c|c|c|}
\hline \multirow{2}{*}{ Herbicida $^{1 /}$} & \multicolumn{4}{|c|}{ Nematicida $^{\underline{1} /}$} \\
\hline & Testemunha & Aldicarb & Carbofuran & Terbufós \\
\hline Testemunha & $0,00 \mathrm{~A} \mathrm{a}$ & $0,00 \mathrm{~A} \mathrm{a}$ & $0,00 \mathrm{~A} \mathrm{a}$ & $0,00 \mathrm{~A} \mathrm{a}$ \\
\hline Tebuthiuron & $1,25 \mathrm{~A} \mathrm{a}$ & $5,00 \mathrm{~A} \mathrm{a}$ & $2,50 \mathrm{~A} \mathrm{a}$ & $3,75 \mathrm{AB}$ \\
\hline Sulfentrazone & $0,00 \mathrm{~A} \mathrm{a}$ & $2,50 \mathrm{~A} \mathrm{a}$ & $1,25 \mathrm{~A} \mathrm{a}$ & $2,50 \mathrm{~A} \mathrm{a}$ \\
\hline Pendimethalin & $3,75 \mathrm{~A} \mathrm{a}$ & $2,50 \mathrm{~A} \mathrm{a}$ & $2,50 \mathrm{~A} \mathrm{a}$ & $5,00 \mathrm{AB} a$ \\
\hline Metribuzin & $1,25 \mathrm{~A} \mathrm{a}$ & $2,50 \mathrm{~A} \mathrm{a}$ & $1,25 \mathrm{~A} \mathrm{a}$ & $3,75 \mathrm{AB}$ \\
\hline Diuron+hexazinone & $1,25 \mathrm{~A} \mathrm{a}$ & $2,50 \mathrm{~A} \mathrm{a}$ & $2,50 \mathrm{~A} \mathrm{a}$ & $3,75 \mathrm{AB}$ \\
\hline Diuron & $1,25 \mathrm{~A} \mathrm{a}$ & $2,50 \mathrm{~A} \mathrm{a}$ & $2,50 \mathrm{~A} \mathrm{a}$ & $3,75 \mathrm{AB}$ \\
\hline Clomazone & $1,25 \mathrm{~A} \mathrm{a}$ & $3,75 \mathrm{~A} \mathrm{a}$ & $2,50 \mathrm{~A} \mathrm{a}$ & $8,75 \mathrm{~B} \mathrm{~b}$ \\
\hline \multirow[t]{2}{*}{ Ametrina } & $0,00 \mathrm{~A} \mathrm{a}$ & $1,25 \mathrm{~A} \mathrm{a}$ & $2,50 \mathrm{~A} \mathrm{a}$ & $3,75 \mathrm{AB}$ a \\
\hline & \multicolumn{2}{|c|}{$\mathrm{DMS}_{\text {(coluna) }}=5,71$} & \multicolumn{2}{|c|}{$\mathrm{DMS}_{(\text {linha) }}=4,71$} \\
\hline
\end{tabular}

1 Médias seguidas de mesma letra, maiúscula na coluna e minúscula na linha, não diferem entre si pelo teste de Tukey a 5\% de significância.

Vale ressaltar que foram encontradas altas infestações de nematóides nas raízes da canade-açúcar e no solo da área experimental durante o experimento (Tabela 7), condição que pode ter interferido nos dados de produtividade, sobretudo reduzindo a produção das parcelas que foram mantidas sem aplicação de nematicidas. Como se pôde perceber, os sintomas de fitotoxicidade diminuíram em relação às avaliações iniciais; contudo, em todos os tratamentos foi observada a recuperação satisfatória das plantas, pois, por ocasião da colheita das parcelas, os valores pesados e extrapolados para toneladas por hectare não mostraram diferenças significativas (Tabela 8).
Ainda, a elevada variabilidade experimental obtida na área também pode ter contribuído para a inexistência de diferenças significativas entre os tratamentos.

Os dados deste trabalho estão em concordância com os de Negrisoli (2001) e Negrisoli et al. (2004), que não observaram diferenças de rendimento significativas entre os tratamentos administrados. Por sua vez, DinardoMiranda et al. (2001b) concluíram que a interação entre terbufós e tebuthiuron resultou em quebra significativa de produtividade agrícola (cerca de $22 \mathrm{t} \mathrm{ha}^{-1}$ a menos em relação à média dos outros tratamentos). 
Tabela 7 - Níveis populacionais de nematóides encontrados nas raízes da cultura da cana-de-açúcar e no solo da área experimental, aos 150 e 210 DAP. Piracicaba, 2003/04

\begin{tabular}{|c|c|c|c|c|c|c|c|c|}
\hline \multirow{3}{*}{ Espécie } & \multicolumn{8}{|c|}{ População de nematóides } \\
\hline & \multicolumn{2}{|c|}{ Testemunha } & \multicolumn{2}{|c|}{ Terbufós } & \multicolumn{2}{|c|}{ Carbofuran } & \multicolumn{2}{|c|}{ Aldicarb } \\
\hline & $\mathrm{R}^{\frac{1}{1}}$ & $\mathrm{~S}^{2 /}$ & $\mathrm{R}$ & $\mathrm{S}$ & $\mathrm{R}$ & $\mathrm{S}$ & $\mathrm{R}$ & $\mathrm{S}$ \\
\hline \multicolumn{9}{|c|}{$150 \mathrm{DAP}$} \\
\hline Meloidogyne incognita & 100 & 0 & 0 & $\overline{0}$ & 0 & 0 & 0 & $\overline{0}$ \\
\hline Pratylenchus zeae & 2.400 & 700 & 1.200 & 600 & 2.000 & 200 & 1.500 & 500 \\
\hline Helicotylenchus & 0 & 100 & 0 & 0 & 0 & 400 & 0 & 0 \\
\hline Paratrichodorus & 0 & 100 & 0 & 0 & 0 & 0 & 0 & 0 \\
\hline \multicolumn{9}{|c|}{210 DAP } \\
\hline Meloidogyne incognita & 1.200 & 2.100 & 0 & 0 & 0 & 0 & 0 & 0 \\
\hline Pratylenchus zeae & 2.800 & 1.500 & 1.500 & 200 & 5.000 & 1.300 & 2.300 & 400 \\
\hline Helicotylenchus & 0 & 680 & 0 & 0 & 0 & 1500 & 0 & 200 \\
\hline Paratrichodorus & 0 & 0 & 0 & 0 & 0 & 0 & 0 & 0 \\
\hline
\end{tabular}

Fonte: ANNA - Análises Nematológicas na Agricultura. ${ }^{1 /} \mathrm{R}-\operatorname{raiz}(50 \mathrm{~g}) ;{ }^{1 /} \mathrm{S}-\operatorname{solo}(1000 \mathrm{~mL}) ;{ }^{3 /}$ Nível populacional: alto.

Tabela 8 - Rendimento $\left(\mathrm{t} \mathrm{ha}^{-1}\right)$ e parâmetros tecnológicos observados na combinação dos tratamentos herbicidas e nematicidas na cana-de-açúcar. Piracicaba, 2003/04

\begin{tabular}{|c|c|c|c|c|c|c|}
\hline Nematicida & Herbicida & $\mathrm{t} \mathrm{ha}^{-1}$ & BRIX & POL (\%) & FIBRA $(\%)$ & ATR (\%) \\
\hline \multirow{9}{*}{ Testemunha } & Testemunha & 123,75 & 19,30 & 14,37 & 11,00 & 138,36 \\
\hline & Tebuthiuron & 96,50 & 19,30 & 14,52 & 11,12 & 139,45 \\
\hline & Sulfentrazone & 113,25 & 19,28 & 14,31 & 11,47 & 137,68 \\
\hline & Pendimethalin & 104,50 & 19,75 & 14,87 & 11,38 & 142,58 \\
\hline & Metribuzin & 98,00 & 19,23 & 14,39 & 11,16 & 138,33 \\
\hline & Diuron+hexazinone & 121,50 & 18,68 & 14,08 & 10,94 & 135,43 \\
\hline & Diuron & 123,50 & 19,28 & 14,45 & 10,99 & 138,92 \\
\hline & Clomazone & 101,50 & 19,40 & 14,60 & 11,21 & 140,15 \\
\hline & Ametrina & 118,50 & 19,60 & 14,59 & 11,39 & 140,23 \\
\hline \multirow{9}{*}{ Aldicarb } & Testemunha & 119,75 & 18,95 & 14,73 & 11,03 & 140,71 \\
\hline & Tebuthiuron & 113,00 & 19,08 & 14,41 & 10,84 & 138,45 \\
\hline & Sulfentrazone & 129,00 & 19,40 & 14,73 & 10,76 & 141,37 \\
\hline & Pendimethalin & 132,50 & 19,63 & 14,64 & 10,85 & 140,91 \\
\hline & Metribuzin & 125,50 & 19,53 & 14,97 & 10,88 & 143,31 \\
\hline & Diuron+hexazinone & 136,00 & 19,38 & 14,77 & 10,96 & 141,57 \\
\hline & Diuron & 118,50 & 19,30 & 14,70 & 10,79 & 141,00 \\
\hline & Clomazone & 130,25 & 19,40 & 14,75 & 10,97 & 141,41 \\
\hline & Ametrina & 104,25 & 19,30 & 14,60 & 10,79 & 140,23 \\
\hline \multirow{9}{*}{ Carbofuran } & Testemunha & 112,75 & 19,48 & 14,69 & 10,86 & 141,12 \\
\hline & Tebuthiuron & 128,00 & 19,58 & 15,07 & 10,51 & 144,28 \\
\hline & Sulfentrazone & $\begin{array}{l}118,00 \\
118,00\end{array}$ & 19,50 & 14,63 & 11,17 & 140,51 \\
\hline & Pendimethalin & 139,00 & 19,65 & 14,73 & 11,05 & 141,55 \\
\hline & Metribuzin & 129,25 & 19,40 & 14,71 & 10,63 & 141,26 \\
\hline & Diuron+hexazinone & 139,00 & 19,28 & 14,63 & 10,82 & 140,41 \\
\hline & Diuron & 124,00 & 19,65 & 14,98 & 11,07 & 143,41 \\
\hline & Clomazone & 123,50 & 19,13 & 14,60 & 10,87 & 140,01 \\
\hline & Ametrina & 125,00 & 19,55 & 14,73 & 11,14 & 141,40 \\
\hline \multirow{9}{*}{ Terbufós } & Testemunha & 128,50 & 19,88 & 15,18 & 10,69 & 145,45 \\
\hline & Tebuthiuron & 113,75 & 19,33 & 14,71 & 10,72 & 141,14 \\
\hline & Sulfentrazone & 112,75 & 19,38 & 14,65 & 10,96 & 140,65 \\
\hline & Pendimethalin & 137,00 & 199,05 & 14,29 & 10,81 & 137,59 \\
\hline & Metribuzin & 137,00 & 19,60 & 14,73 & 11,51 & 141,25 \\
\hline & Diuron+hexazinone & 113,75 & 19,28 & 14,68 & 10,85 & 140,81 \\
\hline & Diuron & 134,25 & 19,68 & 14,89 & 10,90 & 142,87 \\
\hline & Clomazone & 114,25 & 19,35 & 14,46 & 10,94 & 139,11 \\
\hline & Ametrina & 127,00 & 19,18 & 14,30 & 11,23 & 137,61 \\
\hline \multirow{3}{*}{ Valores de F } & Herbicidas $(\mathrm{H})$ & $0,56^{\mathrm{NS}}$ & $0,66^{\mathrm{NS}}$ & $0,84^{\mathrm{NS}}$ & $0,67^{\mathrm{NS}}$ & $0,82^{\mathrm{NS}}$ \\
\hline & Nematicidas (N) & $0,39^{\mathrm{NS}}$ & $0,95^{\mathrm{NS}}$ & $0,78^{\mathrm{NS}}$ & $0,13^{\mathrm{NS}}$ & $0,79^{\mathrm{NS}}$ \\
\hline & $\mathrm{N} \times \mathrm{H}$ & 0,65 Ns" & $0,53^{\mathrm{NS}}$ & $0,40^{\mathrm{Ns}}$ & $0,90^{\mathrm{Ns}}$ & $0,38^{\mathrm{Ns}}$ \\
\hline \multirow{2}{*}{$\mathrm{CV}(\%)$} & Herbicidas & 18,20 & 2,60 & 3,00 & 4,59 & 2,73 \\
\hline & Nematicidas & 28,85 & 7,08 & 8,45 & 3,73 & 7,94 \\
\hline
\end{tabular}

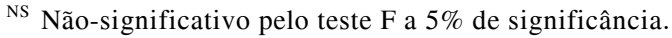


Ainda, pode-se ver que os tratamentos também não interferiram de forma significativa nas características tecnológicas da canade-açúcar (Tabela 7). Negrisoli (2001) concluiu que os herbicidas tebuthiuron, ametrina, sulfentrazone, metribuzin e clomazone aplicados em associação com aldicarb e carbofuran também não interferiram negativamente na qualidade tecnológica da variedade RB 855113.

Assim, com a realização deste experimento, pôde-se observar que os sintomas de fitotoxicidade oriundos da interação de herbicidas e nematicidas foram agudos, com os casos mais evidentes se originando da associação dos nematicidas com clomazone, pendimethalin e tebuthiuron. Contudo, os danos fitotóxicos não se refletiram em perdas significativas de rendimento ou de qualidade de colmos, fato esse que pode ter sido influenciado pela capacidade de recuperação de injúrias apresentada pela variedade RB 867515, pela ocorrência de altas infestações de nematóides ou pela elevada variabilidade experimental.

\section{LITERATURA CITADA}

AHRENS, W. H. Enhancement of soybean (Glycine max) injury and weed control by thifensulfuron-insecticide mixtures. Weed Technol., v. 4, n. 3, p. 524-528, 1990.

BAERG, R. J.; BARRET, M. Insecticide modifications of cytochrome P450 mediated herbicide metabolism. Proc. North Cent. Weed Sci. Soc., v. 48, p. 70, 1993.

BLANCO, G. H. et al. Fitotoxicidade em cana-de-açúcar causada pela interação de inseticida e herbicida residual. O Biológico, v. 46, n. 10, p. 235-240, 1980.

DINARDO-MIRANDA, L. L. et al. Efeitos da interação entre nematicidas e herbicidas em cana-de-açúcar. Nematol. Bras., v. 25, n. 2, p. 197-203, 2001a.

DINARDO-MIRANDA, L. L.; MENEGATTI, C. C.; PIVETTA, J. P. Eficiência de nematicidas aplicados no plantio da cana-de-açúcar. Nematol. Bras., v. 25, n. 2, p. 171-174, 2001b.

DINARDO-MIRANDA, L. L.; FERRAZ, L. C. C. B. Patogenicidade de Pratylenchus brachyurus e P. zeae a duas variedades de cana-de-açúcar. Nematol. Bras., v. 15, n. 1, p. 9-16, 1991.

HARTZLER, B.; PRINGNITZ, B.; OWEN, M. Interactions between ALS-herbicides and organophosphate insecticides. Disponível em: <http://www.ipm.iastate.edu/ $\mathrm{ipm} / \mathrm{icm} /$ 2000/5-22-2000/interaction.html>. Acesso em: 17 de dezembro de 2003.

Planta Daninha, Viçosa-MG, v. 24, n. 2, p. 371-378, 2006
HINZ, J. R. R.; OWEN, M. D. K.; BARRETT, M. Nicosulfuron, primisulfuron, and bentazon hydroxylation by corn (Zea mays), woolly cupgrass (Eriochloa villosa), and shattercane (Sorghum bicolor) cytochrome P-450. Weed Sci., v. 45, p. 474-480, 1997.

KÖEPPEN, W. Climatologia: con un estudio de los climas de la tierra. México: Fondo de Cultura Económica, 1948. 478 p.

LÓPEZ-OVEJERO, R. F. et al. Manejo de plantas daninhas na cultura do milho. In: FANCELLI, A. L.; DOURADO NETO, D. Milho: estratégias de manejo para alta produtividade. Piracicaba: Escola Superior de Agricultura "Luiz de Queiroz", 2003. p. 47-79.

LORENZI, H. Plantas daninhas na cultura da cana-deaçúcar: Plantas daninhas na lavoura do nordeste brasileiro. In: ENCONTRO TÉCNICO GOAL, CANA-DE-AÇÚCAR, 4., 1995, Recife. Anais... Recife: 1995.

LORENZI, H. Plantas daninhas e seu controle na cultura da cana-de-açúcar. In: SEMINÁRIO DE TECNOLOGIA AGRONÔMICA, 4., 1988, Piracicaba. Anais... São Paulo: COOPERSUCAR, 1988. p. 281-301.

NEGRISOLI, E. et al. Seletividade de herbicidas aplicados em pré-emergência na cultura de cana-de-açúcar tratada com nematicidas. Planta Daninha, v. 22, n. 4, p. 567-575, 2004.

NEGRISOLI, E. Seletividade de herbicidas aplicados em pré-emergência e associados à nematicidas, à cultura da cana-de-açúcar cultivar RB-855113. 2001. $48 \mathrm{f}$. Dissertação (Mestrado em Agronomia) - Universidade Estadual Paulista "Julio de Mesquita Filho", Botucatu, 2003.

NOVARETTI, W. R. T. Efeitos de diferentes níveis de populações iniciais de Meloidogyne javanica em duas variedades de cana-de-açúcar (Saccharum spp.) cultivadas no Estado de São Paulo. 1981. 100 f. Dissertação (Mestrado em Agronomia) - Escola Superior de Agricultura "Luiz de Queiroz", Piracicaba, 1981.

PROCÓPIO, S. O.; SILVA, A. A.; VARGAS, L. Manejo e controle de plantas daninhas em cana-de-açúcar. In: VARGAS, L.; ROMAN, E. S. (Eds.). Manual de manejo e controle de plantas daninhas. Bento Gonçalves: Embrapa Uva e Vinho, 2004. p. 397-452.

SIMINSZKY, B.; CORBIN, F. T.; SHELDON, Y. Nicosulfuron resistance and metabolism in terbufos- and naphthalic anhydride-treated corn. Weed Sci., v. 43, p. 163-168, 1995.

VELINI, E. D.; OSIPE, R.; GAZZIERO, D. L. P. Procedimentos para a instalação e análise de experimentos com herbicidas. Londrina: Sociedade Brasileira da Ciência das Plantas Daninhas, 1995. 21 p. 